\title{
Prospective Randomized Study of Doxorubicin-Eluting-Bead Embolization in the Treatment of Hepatocellular Carcinoma: Results of the PRECISION V Study
}

\author{
Johannes Lammer • Katarina Malagari - Thomas Vogl • Frank Pilleul • Alban Denys • \\ Anthony Watkinson • Michael Pitton · Geraldine Sergent · Thomas Pfammatter • \\ Sylvain Terraz • Yves Benhamou - Yves Avajon - Thomas Gruenberger • Maria Pomoni • \\ Herbert Langenberger • Marcus Schuchmann - Jerome Dumortier • Christian Mueller • \\ Patrick Chevallier · Riccardo Lencioni - On Behalf of the PRECISION V Investigators
}

Received: 9 June 2009/Accepted: 21 August 2009/Published online: 12 November 2009

(C) Springer Science+Business Media, LLC and the Cardiovascular and Interventional Radiological Society of Europe (CIRSE) 2009

\begin{abstract}
Transcatheter arterial chemoembolization (TACE) offers a survival benefit to patients with intermediate hepatocellular carcinoma (HCC). A widely accepted TACE regimen includes administration of doxorubicin-oil emulsion followed by gelatine sponge-conventional TACE. Recently, a drug-eluting bead (DC Bead ${ }^{\circledR}$ ) has been developed to enhance tumor drug delivery and reduce systemic
\end{abstract}

The authors represent the PRECISION V Study Collaborators.

The detailed information about the PRECISION V Study collaborators are given in Appendix 1.

J. Lammer $(\bowtie) \cdot H$. Langenberger

Cardiovascular and Interventional Radiology, Medical

University Vienna, Waehringer Guertel 18-20, 1090 Vienna,

Austria

e-mail: johannes.lammer@meduniwien.ac.at

K. Malagari · M. Pomoni

University of Athens, Athens, Greece

T. $\operatorname{Vog} 1$

Goethe University, Frankfurt, Germany

F. Pilleul · J. Dumortier

Hospices Civils de Lyon, CHU, Hopital Edouard Herriot,

Lyon, France

A. Denys

CHU Vaudois, Lausanne, Switzerland

\section{A. Watkinson}

The Peninsula Medical School, Royal Devon and Exeter

Hospital, Exeter, UK

M. Pitton · M. Schuchmann

Guttenberg University, Mainz, Germany availability. This randomized trial compares conventional TACE (cTACE) with TACE with DC Bead for the treatment of cirrhotic patients with HCC. Two hundred twelve patients with Child-Pugh $\mathrm{A} / \mathrm{B}$ cirrhosis and large and/or multinodular, unresectable, N0, M0 HCCs were randomized to receive TACE with DC Bead loaded with doxorubicin or cTACE with doxorubicin. Randomization was stratified according to Child-Pugh status (A/B), performance status (ECOG 0/1), bilobar disease (yes/no), and prior curative treatment (yes/ no). The primary endpoint was tumor response (EASL) at 6 months following independent, blinded review of MRI studies. The drug-eluting bead group showed higher rates of

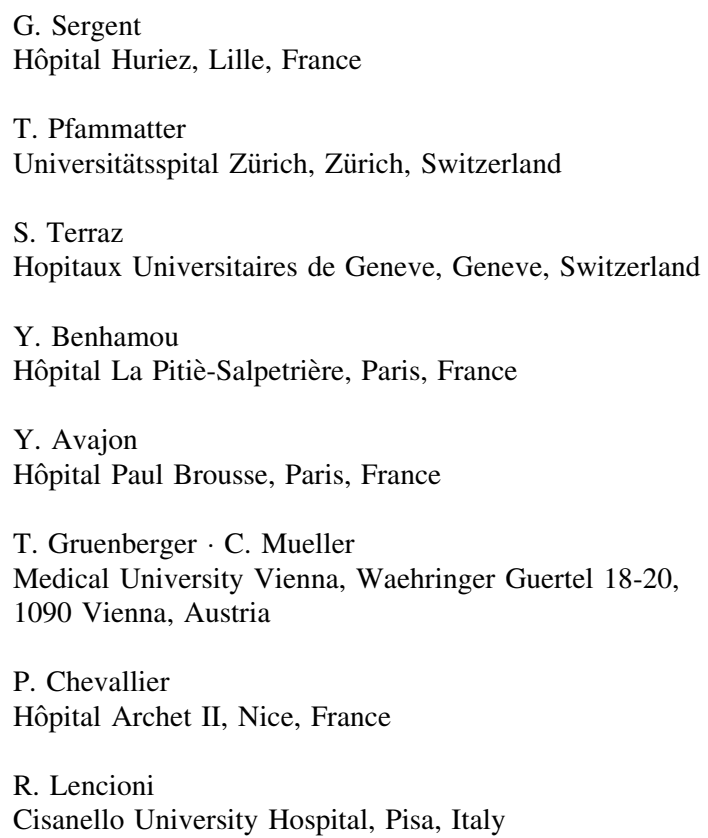


complete response, objective response, and disease control compared with the cTACE group ( $27 \%$ vs. $22 \%, 52 \%$ vs. $44 \%$, and $63 \%$ vs. $52 \%$, respectively). The hypothesis of superiority was not met (one-sided $P=0.11$ ). However, patients with Child-Pugh B, ECOG 1, bilobar disease, and recurrent disease showed a significant increase in objective response $(P=0.038)$ compared to cTACE. DC Bead was associated with improved tolerability, with a significant reduction in serious liver toxicity $(P<0.001)$ and a significantly lower rate of doxorubicin-related side effects $(P=0.0001)$. TACE with DC Bead and doxorubicin is safe and effective in the treatment of HCC and offers a benefit to patients with more advanced disease.

Keywords Chemoembolization .

Hepatocellular carcinoma · Doxorubicin .

Drug-eluting beads

\section{Introduction}

Hepatocellular carcinoma (HCC) is an increasingly common tumor with a poor prognosis and limited systemic treatment options; approximately $80 \%$ of patients die within a year of diagnosis. In men, it is the fifth most common cancer worldwide and the third leading cause of cancer-related death [1, 2].

Barcelona Clinic Liver Cancer (BCLC) tumor staging $[3,4]$ combines the stage of liver disease, tumor stage, clinical performance, and treatment options and is endorsed by the European Association for the Study of Liver Disease (EASL) and the American Association for the Study of Liver Disease (AASLD) [5]. In countries where no systematic screening of cirrhotic patients is performed, 50$75 \%$ are diagnosed when $\mathrm{HCC}$ is at an advanced stage (BCLC Stage C, Child-Pugh A/B, cancer symptoms present, and/or vascular invasion and extrahepatic spread) [6]. Such patients are precluded from surgery, and for many years doxorubicin had been used for systemic treatment [5], albeit without a proven survival benefit [7]. Thus, there is interest in new antiangiogenic agents. Recent data have established Sorafenib as the preferred systemic therapy for advanced HCC [8]. For unresectable intermediate-stage HCC (BCLC Stage B, Child-Pugh A/B, with large or multifocal $\mathrm{HCC}$, no vascular invasion or extrahepatic spread), the current standard treatment is transarterial chemoembolization (TACE) [6].

TACE involves the periodic injection of a chemotherapeutic agent, mixed with embolic material, administered selectively into the feeding arteries of the tumor to potentially obtain higher intratumor drug concentrations compared to intravenous therapy, with occlusion of the blood vessel causing infarction and necrosis [9]. In HCC patients, TACE has achieved partial responses in up to $62 \%$ of patients, as well as significantly delayed tumor progression and vascular invasion [10-16]. Llovet et al. were the first to show a statistically significant benefit in survival for chemoembolization using doxorubicin (50$75 \mathrm{mg} / \mathrm{m}^{2}$ ) and Gelfoam compared with best supportive care (BSC) [16]. Although a survival benefit of TACE over symptomatic treatment or systematic chemotherapy was demonstrated in a meta-analysis of randomized controlled trials, overall survival at 3 years remained low $(<30 \%)$ for intermediate HCC patients [17]. A further review failed to demonstrate either a survival difference between TACE and embolization alone or superiority of one chemotherapeutic agent over another [18]. Post-TACE complications, e.g., acute liver or renal failure, encephalopathy, ascites, and upper gastrointestinal bleeding, may be severe [18]. There is therefore a requirement for treatment regimens that improve response rates and survival, while reducing the risk of post-TACE complications.

The DC Bead (Biocompatibles UK Ltd.) is a novel drug delivery embolization system, comprising biocompatible, nonresorbable hydrogel beads capable of being loaded with anthracyclin derivatives such as doxorubicin [19, 20], the most widely used chemotherapeutic for the treatment of HCC. Preclinical [21, 22] and clinical [23, 24] studies have shown that TACE with DC Bead results in higher tumor concentrations and lower systemic concentrations of doxorubicin compared to intra-arterial doxorubicin and conventional TACE. Phase I/II studies in HCC have demonstrated promising efficacy with low toxicity [23-26].

This phase II study is the first comparative study designed to evaluate the safety and efficacy of DC Bead with doxorubicin in the treatment of HCC in comparison with conventional TACE (cTACE), the current standard treatment [18].

\section{Materials and Methods}

\section{Patients}

Patients aged $>18$ years with $\mathrm{HCC}$ unsuitable for resection or percutaneous ablation, (BCLC A/B, without portal invasion or extrahepatic spread) were eligible for the study. Eligibility criteria also included: no previous chemotherapy, radiotherapy or transarterial embolization (with or without chemotherapy), a confirmed diagnosis of HCC according to EASL, an Eastern Cooperative Oncology Group (ECOG) performance status of 0 or 1 , and preserved liver function (Child-Pugh Class A or B). Patients were excluded if they had another primary tumor, advanced liver 
disease (bilirubin levels $>3 \mathrm{mg} / \mathrm{dl}$, AST or ALT $>5 \times$ upper limit of normal or $>250 \mathrm{U} / \mathrm{l})$, advanced tumoral disease (vascular invasion or extrahepatic spread, or diffuse HCC, defined as $>50 \%$ liver involvement), or contraindications for doxorubicin administration.

All patients provided written informed consent. The study was performed in accordance with the Declaration of Helsinki, the International Conference on Harmonization Guideline on Good Clinical Practice, and relevant local laws and regulations. Ethics committee approval was obtained. Independent Data and Safety Monitoring Committees were established to monitor efficacy and safety data. The study was registered at www.clinicaltrials.gov (NCT00261378), conducted according to the HCC Clinical Trial Design guidelines [27], and reported according to CONSORT recommendations [28, 29].

\section{Study Design}

This was an international, multicenter, prospective, randomized, single-blind, phase II study. Patients were randomized (1:1) to receive doxorubicin via TACE with DC Bead or conventional TACE (cTACE). Randomization was centralized, with stratification factors of Child-Pugh class (A/B), ECOG performance status (0/1), prior curative (resection or percutaneous ablation) treatment (yes/no), and bilobar disease (yes/no), representing more advanced disease. Randomized treatment allocation was predetermined by an independent statistician and used a randomized permuted block design to ensure that, at the conclusion of the study treatment, group sizes were similar both overall and for each level of stratification factor. The randomization was integrated into the web-based Case Report Form after screening.

Dose selection was based on cTACE treatment protocols of doxorubicin, $50-75 \mathrm{mg} / \mathrm{m}^{2}$. Patients typically receive between 100 and $150 \mathrm{mg}$ in a single treatment session. In two previous dose escalation studies [23, 24], DC Bead loaded with doxorubicin in the range from 25 to $150 \mathrm{mg}$ was shown to be safe and effective even at the highest dose of $150 \mathrm{mg}$.

In this study, patients in the DC Bead group received $4 \mathrm{ml}$ DC Bead (1 vial of 300-500 $\mu \mathrm{m}$ first, followed by 1 vial of 500-700 $\mu \mathrm{m}$ ) loaded with doxorubicin (150 mg per procedure) mixed with nonionic contrast medium. Lipiodol (iodinated poppy seed oil; Guerbet, France) was not used. No dose adjustment was made for bilirubin concentration or body surface area. In the cTACE group, patients received an intra-arterial injection of an emulsion of doxorubicin (50-75 $\mathrm{m}^{2}$ to a maximum of $150 \mathrm{mg}$, adjusted for bilirubin concentration and body surface area) in lipiodol followed by particle embolization with an embolic agent of the investigator's choice (Gelfoam particles, Embosphere, Contour SE, Bead Block, PVA particles). In both treatment groups, patients were treated at 2-monhtly intervals [25, 26], received a maximum of three chemoembolizations (at baseline, 2 months, and 4 months), and were followed for 6 months. For patients with bilobar disease who could not be treated superselectively in a single treatment, a second embolization, of the alternative lobe, was performed within 3 weeks of the first procedure (procedure 1B) provided that there was no contraindication due to systemic toxicity and/or clinical performance. Reasons for discontinuation of treatment included ineligibility prior to chemoembolization, progressive disease, and severe systemic toxicity.

In both treatment arms, catheterization was performed via a femoral artery, and superselective embolization of the hepatic artery branches feeding the tumor was performed. The embolization endpoint was defined as stasis in the second- or third-order branches of the right or left hepatic artery. A microcatheter could be used to select a branch feeding the tumor.

Tumor response according to EASL criteria was evaluated by magnetic resonance imaging (MRI) performed at baseline and 1, 3, and 6 months (Fig. 1). MRI was performed on 1.5-T scanners using a spoiled gradient-echo T1-weighted sequence and fast spin-echo T2-weighted sequence with fat suppression. A dynamic multiphasic, contrast-enhanced, spoiled gradient-echo T1-weighted sequence with arterial, portal, equilibrium, and delayed phase was performed. MRI scans were assessed independently by two assessors blinded to treatment allocation (followed by adjudication in case of disagreement).

\section{Study Hypothesis and Objective}

The hypothesis was that treatment of HCC with DC Bead is superior to treatment with cTACE. The objective was to evaluate the safety and 6-month tumor response of chemoembolization with DC Bead vs. cTACE.

Outcomes and Procedures/Assessments

\section{Efficacy}

The primary efficacy endpoint was the 6-month tumor response rate, according to the amended EASL response criteria, an accepted method for assessing tumor necrosis following locoregional therapy [30]. According to this amendment, response of target lesions is classified according to the presence and the dimensions of the viable tumor, defined as a tumor that takes up contrast in the 
Fig. 1 Flowchart of patients in the PRECISION V Trial. * For patients with bilobar disease who could not be treated superselectively in a single treatment, a second embolization was performed (procedure 1B) for the alternative lobe within 3 weeks of the first procedure: DC Bead $(n=8)$ vs. cTACE $(n=5)$

\begin{tabular}{|c|c|c|}
\hline & DC Bead ${ }^{\mathrm{TM}}$ Group & cTACE Group \\
\hline Baseline Assessments/Randomization & & \\
\hline Randomised & $\mathrm{N}=102$ & $\mathbf{N}=\mathbf{1 1 0}$ \\
\hline Received Treatment & $\mathrm{N}=93^{*}$ & $\mathrm{~N}=108 *$ \\
\hline Did not receive intervention & $\mathrm{N}=9$ & $\mathbf{N}=\mathbf{2}$ \\
\hline Refused & $N=2$ & $N=0$ \\
\hline Did not meet inclusion criteria & $N=4$ & $N=1$ \\
\hline Progression & $N=1$ & $N=0$ \\
\hline Other & $N=2$ & $N=1$ \\
\hline Discontinued treatment & $\mathrm{N}=27$ & $\mathrm{~N}=\mathbf{4 0}$ \\
\hline Adverse event & $N=12$ & $N=14$ \\
\hline Patient consent withdrawal & $N=3$ & $N=4$ \\
\hline Post consent ineligibility & $N=0$ & $N=1$ \\
\hline Lack of efficacy & $N=2$ & $N=8$ \\
\hline Downstaged & $N=5$ & $N=8$ \\
\hline Lost to Follow-up & $N=2$ & $N=1$ \\
\hline Death & $N=0$ & $N=3$ \\
\hline Other & $N=3$ & $N=1$ \\
\hline Analysed Population & $\mathrm{N}=93$ & $\mathrm{~N}=108$ \\
\hline
\end{tabular}

arterial phase of radiologic imaging. The primary readers manually traced viable tumor on MRI slices obtained at baseline and after treatment, and computer calculations were made to quantify percentage changes in viable tumor volume and therefore to define the response of target lesions. The emergence of one or more new lesions was considered evidence of progression in the overall patient response assessment, regardless of the response obtained in target lesions. Objective response rate (OR) was defined as complete response plus partial response, and disease control rate as OR plus stable disease.

\section{Safety}

The primary safety endpoint was the incidence of treatment-related serious adverse events (SAEs) occurring within 30 days of a treatment procedure. Secondary safety outcomes included the incidence and severity of adverse events (AEs) and SAEs, liver function parameters, laboratory abnormalities, and cardiac function (ejection fraction).

\section{Statistical Analysis}

The study aimed to reject with $80 \%$ power, at a one-sided significance level of $\alpha=0.025$, the null hypothesis $\mathrm{H} 0$, i.e., $\pi \mathrm{DC} \quad \mathrm{Bead}=\pi \mathrm{cTACE}$ against the alternative hypothesis, H1, i.e., $\pi \mathrm{DC}$ Bead $>\pi \mathrm{cTACE}$, where $\pi$ denotes the rate of patients in the respective treatment arm with an objective tumor response at 6 months. The study was conducted using an adaptive three-stage group sequential test design within the $\Delta$ class of critical values from Wang and Tsiatis [31]. Assuming objective tumor response rates of 55\% (DC Bead) and 35\% (cTACE), and a total $n=200$, the statistical power was approximately $81.3 \%$. Interim analyses were conducted independently and results kept confidential until completion of the trial. Following each interim analysis, the Data Monitoring Committee made recommendations to continue, modify, or stop the trial, based on interim analysis results (stopping criteria at $n 1=60$, boundary $P$-value $=0.00015 ; n 2=40$, boundary $P$-value $=0.00258 ; n 3=100$, boundary $P$ value $=0.02396$ ). 
The primary analysis of efficacy was based on the Modified Intention-to-Treat (MITT) population, defined as all randomized patients who received at least one chemoembolization; this also defined the safety population. The primary safety endpoint was analyzed using the chi-square test and all safety data are presented descriptively.

All other group comparisons were supportive in nature and the secondary efficacy endpoints were analyzed descriptively using appropriate statistical methods for each endpoint. Where statistical modeling was used, baseline stratification factors were included. All endpoints were also presented using descriptive statistical methods and all statistical testing was two-sided at the 5\% level of significance. The preplanned analyses of the subgroup of more advanced patients (Child-Pugh B, ECOG 1, bilobar disease, recurrent disease) revealed a clinically relevant trend toward a higher OR rate for DC Bead over cTACE. Supplementary post hoc analyses focused on treatment response and safety were therefore undertaken. The frequency of doxorubicin-related AEs, and of overall AEs, and the level of liver toxicity were explored further. These data were hypothesis-generating in nature, and $P$-values should be interpreted in the exploratory sense.

\section{Results}

Patients were enrolled at 19 centers in five countries (France, Germany, Switzerland, Austria, and Greece) between 25 November 2005 and 27 June 2007. A total of 212 patients were randomized to TACE with DC Bead $(n=102)$ or cTACE $(n=110)$. Due to dropouts prior to first treatment, the MITT population included 93 and 108 patients. The last-observation-carried-forward (LOCF) principle was used so that the primary endpoint could be assessed using the full MITT population (Fig. 1). At

Table 1 Patient characteristics, health status, and tumor burden at baseline

\begin{tabular}{|c|c|c|}
\hline Characteristic & DC Bead $(n=93)$ & $\operatorname{cTACE}(n=108)$ \\
\hline Age, years $($ mean $\pm \mathrm{SD})$ & $67.3 \pm 9.1$ & $67.4 \pm 8.8$ \\
\hline Gender (male/female) & $79 / 14$ & $95 / 13$ \\
\hline Etiology of cirrhosis ${ }^{\mathrm{a}}$ (HCV/HBV/alcohol/other $\left.{ }^{\mathrm{b}}\right)$ & $22 / 16 / 43 / 21$ & $18 / 18 / 57 / 25$ \\
\hline \multicolumn{3}{|l|}{ Health status } \\
\hline Prior curative treatment $(\text { no/yes })^{\mathrm{c}}$ & $82 / 11$ & $95 / 13$ \\
\hline Prior surgery ${ }^{\mathrm{d}}$ & $7(7.5 \%)$ & $9(8.3 \%)$ \\
\hline Radiofrequency ablation $^{\mathrm{d}}$ & $5(5.4 \%)$ & $3(2.8 \%)$ \\
\hline Percutaneous ethanol injection $^{\mathrm{d}}$ & $1(1.1 \%)$ & $2(1.9 \%)$ \\
\hline Thermoablation $^{\mathrm{d}}$ & 0 & $2(1.9 \%)$ \\
\hline Bilobar disease $(\text { no/yes })^{c}$ & $52 / 41$ & $63 / 45$ \\
\hline Child-Pugh classification $(\mathrm{A} / \mathrm{B})^{\mathrm{c}}$ & $77 / 16$ & $89 / 19$ \\
\hline ECOG performance status $(0 / 1)^{c}$ & $74 / 19$ & $80 / 28$ \\
\hline Okuda tumor classification (I/II) & $79 / 14$ & $103 / 5$ \\
\hline BCLC classification $(\mathrm{A} / \mathrm{B} / \mathrm{C})^{\mathrm{e}}$ & $24 / 69 / 0$ & $29 / 79 / 0$ \\
\hline Karnofsky performance status $(100 / 90 / 80 / \leq 70)$ & $60 / 27 / 5 / 1$ & $51 / 42 / 10 / 5$ \\
\hline Encephalopathy (no/yes) & $93 / 0$ & $107 / 1$ \\
\hline LVEF, $\%($ mean $\pm \mathrm{SD})$ & $66.2 \pm 8.4$ & $64.3 \pm 8.2$ \\
\hline \multicolumn{3}{|l|}{ Tumor burden } \\
\hline No. of nodules $\left(1 / 1+{ }^{\mathrm{f}} / 2 /\right.$ multinodular $)$ & 28/11/19/35 & $32 / 8 / 18 / 50$ \\
\hline Mean no. of lesions (range) & $2.8(1-20)$ & $3.8(1-50)$ \\
\hline Total sum of diameters of HCC lesions, mm (mean \pm SD) & $88.9 \pm 52.1$ & $89.2 \pm 59.3)$ \\
\hline Mean liver involvement, \% (range) & $16.1(<10-50)$ & $16.1(<10-50)$ \\
\hline
\end{tabular}

${ }^{a}$ Multiple responses per patient were possible

b Autoimmune hepatitis, cryptogenic cirrhosis, haemochromatosis, hepatic cirrhosis due to prolonged cytostatic therapy, hepatic steatosis, hepatitis D, non-cirrhotic and unknown

c Stratification factors

${ }^{\mathrm{d}}$ Type of prior curative treatments: number of patients (\% of patients); multiple different curative treatments possible

e BCLC classification according to tumor stage [32]

${ }^{\mathrm{f}} 1+=1+$ satellite 
baseline there were no major differences between the treatment groups with regard to patient demographics, tumor burden, or health status (Table 1). The majority of patients $(66.7 \%)$ in both groups were considered more advanced, as they met the higher risk criteria for one or more of the four prognostic factors, i.e., Child Pugh B, ECOG 1, bilobar or recurrent disease (63 of 93 DC Bead and 72 of 108 cTACE patients). The mean total dose of doxorubicin administered was higher in the DC Bead group compared with the cTACE group ( $295 \mathrm{vs.} 223 \mathrm{mg}$ ) and in all subgroups. The mean volume of lipiodol administered was $10 \mathrm{ml}$ per treatment in the cTACE group [32]. The numbers of chemoembolizations in each treatment group were similar: 93 and 108 patients, respectively, received a first; $82 \%$ of patients in each group received a second; and $61 \%$ and $57 \%$, respectively, received a third chemoembolization.

\section{Efficacy (Tumor Response-EASL)}

At 6 months, a complete response was achieved in 25 (26.9\%) vs. $24(22.2 \%)$ patients, a partial response in 23 $(24.7 \%)$ vs. $23(21.3 \%)$ patients, and stable disease in 11 (11.8\%) vs. 9 (8.3\%) patients in the DC Bead vs. cTACE arm, respectively. Progressive disease was observed in 30 (32.3\%) vs. 44 (40.7\%) patients, respectively; 4 DC Bead patients and 8 cTACE patients withdrew prior to the first
MRI scan. Reasons for these withdrawals were AEs (four DC Bead and four cTACE), withdrawn consent (two cTACE), and post consent ineligibility (two cTACE). Therefore, the OR rate was $51.6 \%$ vs. $43.5 \%$ in the DC Bead vs. cTACE arm, respectively; the hypothesis of superiority was not met (one-sided $P=0.11$ ) (Fig. 2). The difference between groups in favor of DC Bead was $8.1 \%$ (two-sided 95\% repeated confidence interval (RCI), -4.8 to $22.6 \%$ ). The disease control rates were $63.4 \%$ vs. $51.9 \%$, respectively (two-sided $P=0.11$ ). Boundary $P$-values were not exceeded in the interim analyses.

Supplementary analyses showed that in the $67 \%$ of patients with more advanced disease (Child Pugh B, ECOG 1 , bilobar or recurrent disease), the incidence of OR and disease control rates were statistically higher $(P=0.038$ and $P=0.026$, respectively) in the DC Bead compared with the cTACE group (Fig. 2). The greatest difference in disease control rates between DC Bead and cTACE occurred in the ECOG 1 and Child-Pugh B subgroups (both, 63\% and 32\%, respectively; Fig. 3).

\section{Safety}

There was no statistically significant $(P=0.86)$ difference between treatments for the primary safety endpoint (treatment-related SAEs within 30 days of a procedure): 19 (20.4\%) DC Bead patients experienced 28 events and 21

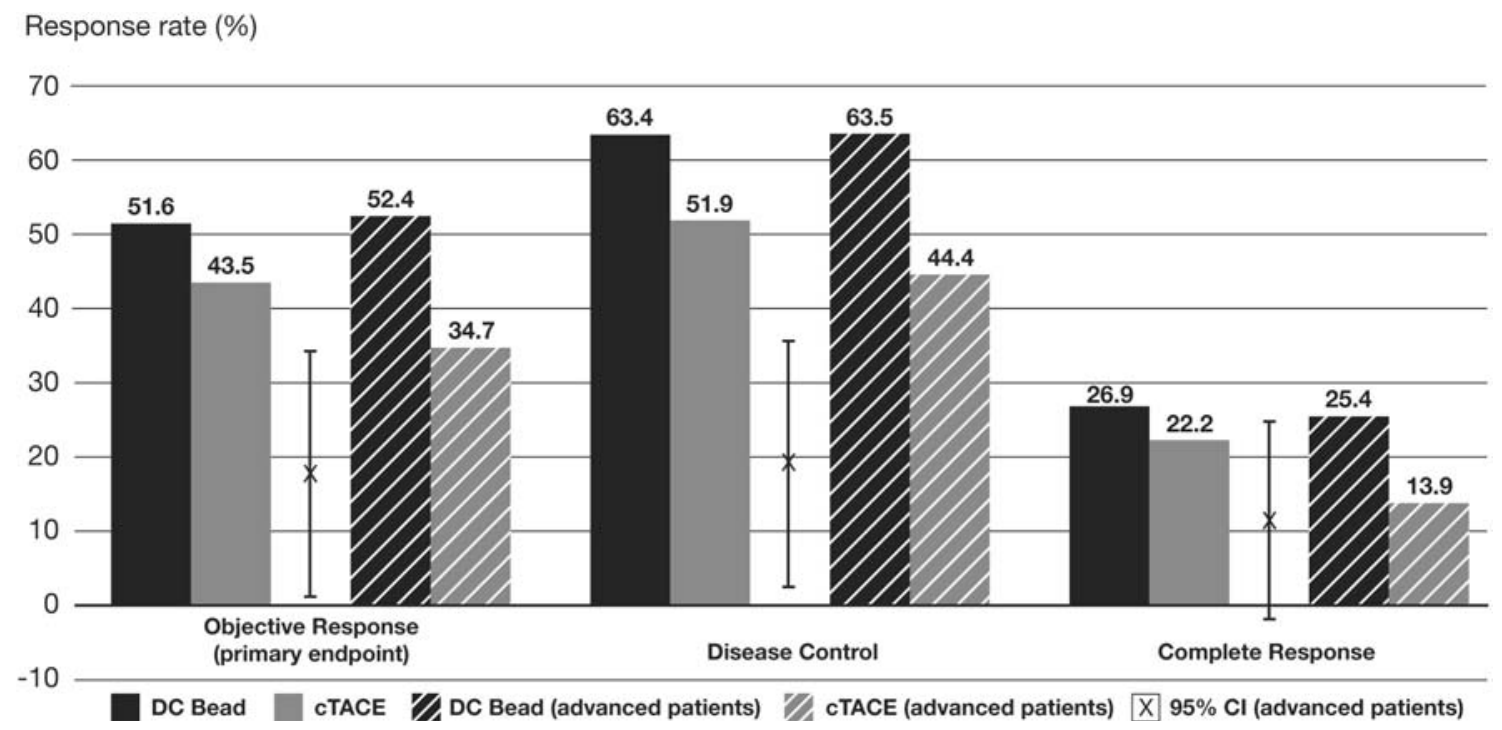

Fig. 2 Tumor response at 6 months (LOCF) (MITT population and advanced patient group $* * *)$. $*$ More advanced disease was at least one of Child-Pugh B, ECOG 1, undergone prior curative treatment (i.e., recurrent disease), and presence of bilobar disease. In accordance with the EASL criteria: complete response (CR) - complete disappearance of all known viable tumor (assessed via uptake of contrast in the arterial phase of the MRI scan) and no new lesions; partial response $(\mathrm{PR})-50 \%$ reduction in viable tumor area of all measurable lesions; stable disease (SD) - all other cases; progressive disease (PD) $-25 \%$ increase in size of one or more measurable lesions or the appearance of new lesions. Objective response (OR) was defined as $\mathrm{CR}+\mathrm{PR}$, and disease control (DC) as $\mathrm{CR}+\mathrm{PR}+\mathrm{SD}$. ** Analysis of advanced patient subgroup: OR rate, $P=0.038$; DC rate, $P=0.026$; CR rate, $P=0.091$ (chi-square analysis) 
Fig. 3 a Complete response, objective response, and disease control rate (cumulative number [\%] of patients) of all patients at 6 months. b Complete response, objective response, and disease control rate (cumulative number [\%] of patients) of patients by stratification factors for advanced disease at baseline
A

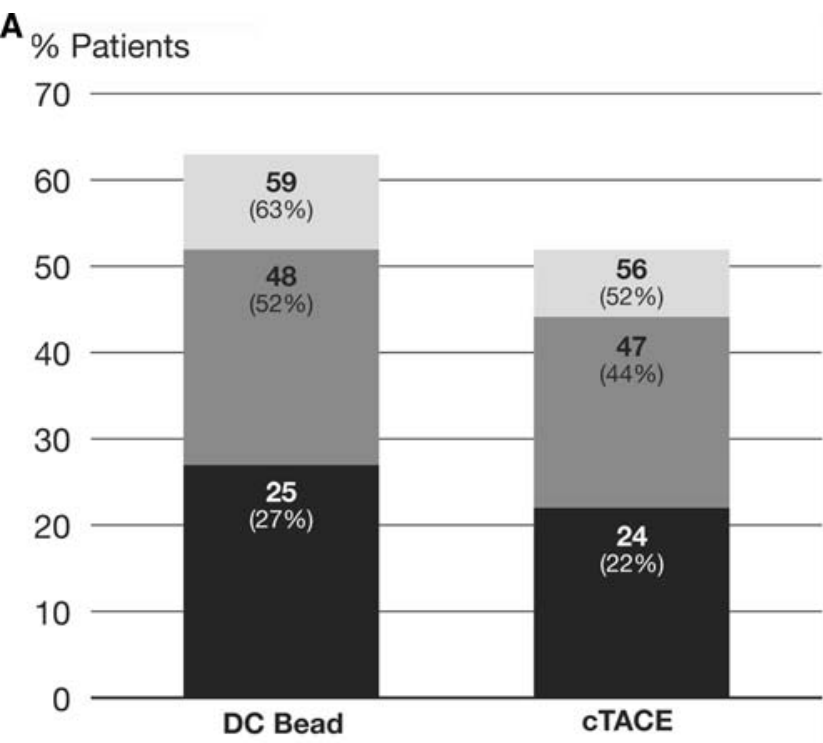

Complete Response

Objective Response

Disease Control

B

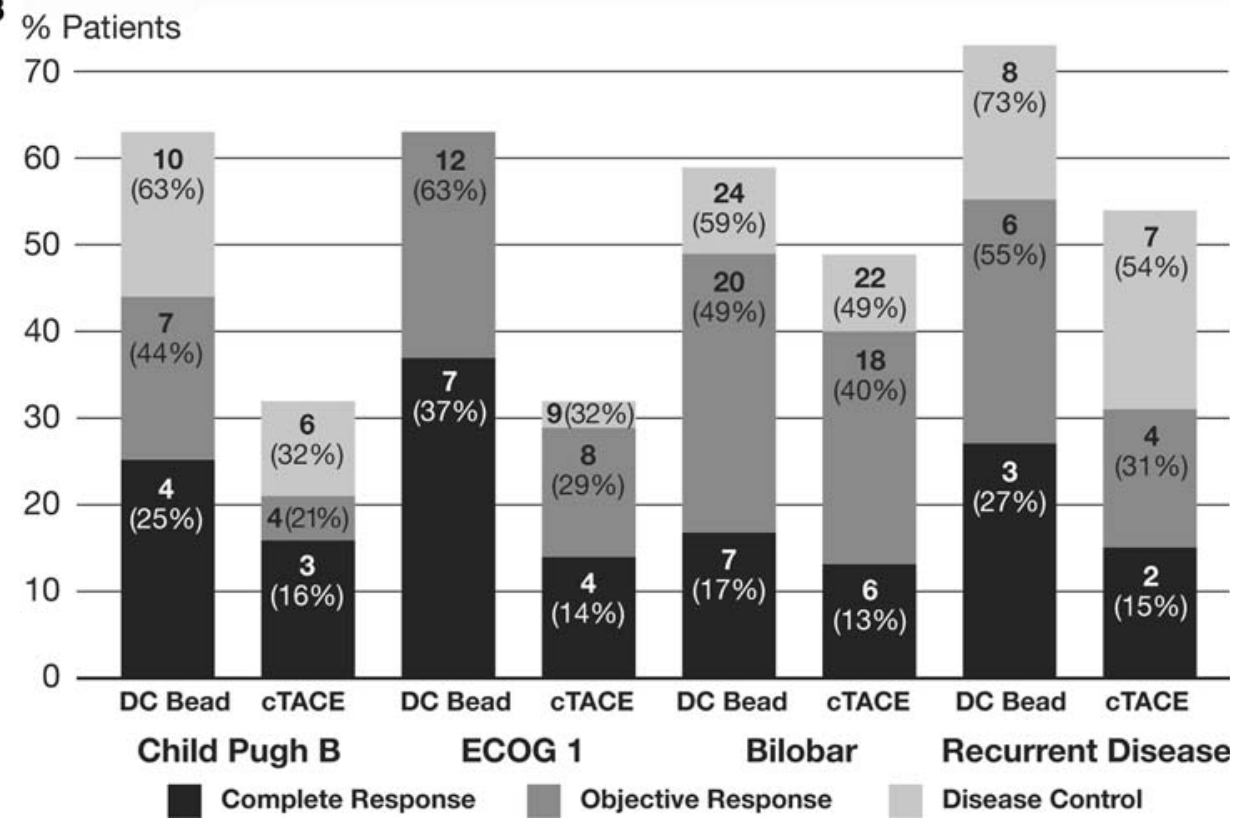

(19.4\%) cTACE patients experienced 24 events. Supplementary post hoc analysis indicated that the incidence of SAEs within 30 days of a procedure was consistently lower in the DC Bead group both for the less advanced and for the more advanced patients based on the four stratification factors (Table 2).

The overall frequency of treatment-emergent AEs (TEAEs) per 100 treatments was lower in the DC Bead compared with the cTACE group, as were treatment-related TEAEs, Southwestern Oncology Group (SWOG) toxicity Grade 3 or 4 TEAEs, Grade 3 or 4 treatment-related TEAEs, and treatment-related SAEs. The majority of TEAEs were mild or moderate in intensity, with a lower frequency of severe events (20.4\% vs. $30.6 \%$ ) reported in DC Bead vs. cTACE patients. The only event with a difference in incidence of $\geq 10 \%$ was alopecia, reported in $2.2 \%$ of DC Bead and $19.4 \%$ of cTACE patients. Serious liver toxicity postchemoembolization was also lower in the DC Bead group. Observed postprocedural increases in the liver enzymes AST and ALT were significantly less in the DC Bead group than in the cTACE group. The mean maximum ALT increase in the DC Bead group was $50 \%$ less than in the cTACE group (95\% CI, 39-65\%; $P<0.001)$ and $41 \%$ less with respect to AST $(95 \% \mathrm{CI}, 46-76 \% ; P<0.001)$ (Fig. 4). Cardiac function (measured by echocardiography, isotopic ventriculography, or MRI) was maintained in the 
Table 2 Incidence of serious adverse events within 30 days of a procedure, by stratification (safety population)

\begin{tabular}{lcclcc}
\hline Stratification factor & \multicolumn{2}{l}{ DC Bead $(n=93)$} & & \multicolumn{2}{c}{ cTACE $(n=108)$} \\
\cline { 2 - 3 } & $\begin{array}{l}\text { No. of } \\
\text { patients/total }\end{array}$ & $\%$ & & $\begin{array}{l}\text { No. of } \\
\text { patients/total }\end{array}$ & $\%$ \\
\hline All patients & $22 / 93$ & 23.7 & & $32 / 108$ & 29.6 \\
Child-Pugh A & $19 / 77$ & 24.7 & & $26 / 89$ & 29.2 \\
Child-Pugh B & $3 / 16$ & 18.8 & $6 / 19$ & 31.6 \\
ECOG 0 & $17 / 74$ & 23.0 & & $23 / 80$ & 28.8 \\
ECOG 1 & $5 / 19$ & 26.3 & $9 / 28$ & 32.1 \\
Unilobar & $12 / 52$ & 23.1 & & $18 / 63$ & 28.6 \\
Bilobar & $10 / 41$ & 24.4 & & $14 / 45$ & 31.1 \\
No prior curative & $19 / 82$ & 23.2 & $28 / 95$ & 29.5 \\
$\quad$ treatments & & & & \\
Recurrent disease & $3 / 11$ & 27.3 & $4 / 13$ & 30.8 \\
\hline
\end{tabular}

Note: Serious adverse events were defined as events (1) resulting in death, (2) that were immediately life-threatening, (3) resulting in permanent or significant disability/incapacity, or (4) requiring or extending inpatient hospitalization or (5) congenital anomaly/birth defects. Analysis of treatment groups overall: chi-square test, $P=0.34$; difference in incidence rates, $-6.0 \% ; 95 \% \mathrm{CI},-18.2$ to 6.2

DC Bead group, whereas there was a deterioration in left ventricular ejection fraction in the cTACE group (DC Bead, $+2.7 \pm 10.1$ percentage points; cTACE, $-1.5 \pm 7.6$ percentage points; $P=0.018$ ). Sixteen deaths were reported during the study (eight in each arm). Of these, two DC Bead and six cTACE patients died within 30 days of a procedure. Four patients died due to disease progression (one DC Bead, three TACE). Other causes of death were liver failure (two in each arm), cardiac events (two DC Bead, one cTACE), infection (one DC Bead, two cTACE), and, in one DC Bead patient, each of GI bleed and unknown cause.

With regard to the systemic side effects of doxorubicin (alopecia, skin discoloration, mucositis, and marrow suppression), post hoc analyses established a significant benefit (estimate of true incidence, $-14.1 \%$; $95 \% \mathrm{CI},-24.7 \%$ to $-3.5 \% ; P=0.012$ ) in favor of DC Bead over cTACE: 12 events in $11(11.8 \%)$ patients vs. 40 events in 28 (25.9\%) patients, respectively. Alopecia, the most commonly occurring event, was almost completely absent in DC Bead patients (1 vs. 23 events). The only DC Bead alopecia event was mild (Grade 1), while in the cTACE arm almost half of the alopecia events (11 events) were of pronounced/ total hair loss (Grade 2). Marrow suppression and mucositis were more common and of greater severity in cTACE compared with DC Bead patients, and skin discoloration occurred in equal numbers (Table 3).Using the assumption of independence of events, the difference in frequencies of doxorubicin-related events was also significant $(P=0.0001)$. The incidence and frequency of post embolization syndrome events were comparable in the treatment groups: 35 events in 23 (24.7\%) DC Bead and 43 events in $28(25.9 \%)$ cTACE patients.

\section{Discussion}

Currently, cTACE is the standard first-line treatment for patients with inoperable and intermediate HCC. Although TACE has been in use for several years, the procedure varies widely between centers, with different drugs (doxorubicin, mitomycin, cisplatin, mixtures), embolic agents, doses, and schedules used. Response rates vary and evidence of a survival benefit, particularly at 3 years, is low [15-18]. In the PRECISION V study, cTACE was designed to reflect the current practice of chemoembolization and was standardized to the use of doxorubicin, lipiodol, and an embolic agent.

The DC Bead is a novel drug delivery embolization system that has been designed to deliver a higher and more sustained release of drug directly into the tumor and a low release of drug into the systemic circulation, with the intention to maximize the drug's effectiveness in terms of response, while significantly reducing its systemic toxicity [22]. Preclinical and clinical studies have demonstrated a higher and prolonged retention of doxorubicin within the tumor after TACE with DC Bead, and lower systemic plasma levels of doxorubicin, compared to cTACE [19-24]. Varela et al. reported significantly lower doxorubicin $\mathrm{C}_{\max }$ and $\mathrm{AUC}$ values in DC Bead patients $(78.97 \pm 38.3 \mathrm{ng} / \mathrm{ml}$ and $662.6 \pm 417.6 \mathrm{ng} / \mathrm{ml} \mathrm{min)}$ than in cTACE patients $(2341.5 \pm 3951.9 \mathrm{ng} / \mathrm{ml}$ and $1812.2 \pm 1093.7 \mathrm{ng} / \mathrm{ml} \mathrm{min}$; $P=0.00002$ and $P=0.001$, respectively)[23]. In another phase I/II clinical study Poon et al. observed low $\mathrm{C}_{\max }$ values $(52.8 \pm 41.5 \mathrm{ng} / \mathrm{ml})$ even at the highest possible loading of doxorubicin $(150 \mathrm{mg})$, with an average half-life of doxorubicin in plasma of $73.5 \pm 22.7 \mathrm{~h}$ [24]. Clinical pilot studies in HCC have demonstrated promising efficacy [23-26, 33].

The current study is the first international, multicenter, randomized study designed to evaluate the safety and efficacy of a drug-eluting bead (DC Bead) compared to cTACE for the treatment of HCC. The 6-month OR rate of $52 \%$ observed following TACE with DC Bead in this study compares well with previously reported OR rates of 44 $82 \%$ in phase I/II studies with DC Bead [23-26]. Although in this study statistical superiority in OR rates compared to cTACE could not be demonstrated, a trend toward higher response rates in all categories (complete response, OR, and disease control) was observed for DC Bead over cTACE. Of particular note was the significant reduction in serious liver toxicity and doxorubicin side effects with DC Bead, despite the higher mean total dose administered, 
Fig. 4 Comparison of treatment groups for-fold changes in liver enzymes by chemoembolization procedure and maximum-fold change across all procedures (mean, 95\% confidence interval $[\mathrm{CI}]$ ). Analysis using $t$-test for logtransformed data; results backtransformed to ratio scale for presentation. Procedure 1B not shown due to small sample

size. a Alanine

aminotransferase (ALT): procedures 1 and 2 and maximum across all procedures, $P<0.001$; procedure $3, P=0.004$. b Aspartate aminotransferase (AST): procedure 1 , $P=0.001 ;$ procedure 2 and maximum across all procedures, $P<0.001$ procedure $3, P=0.06$
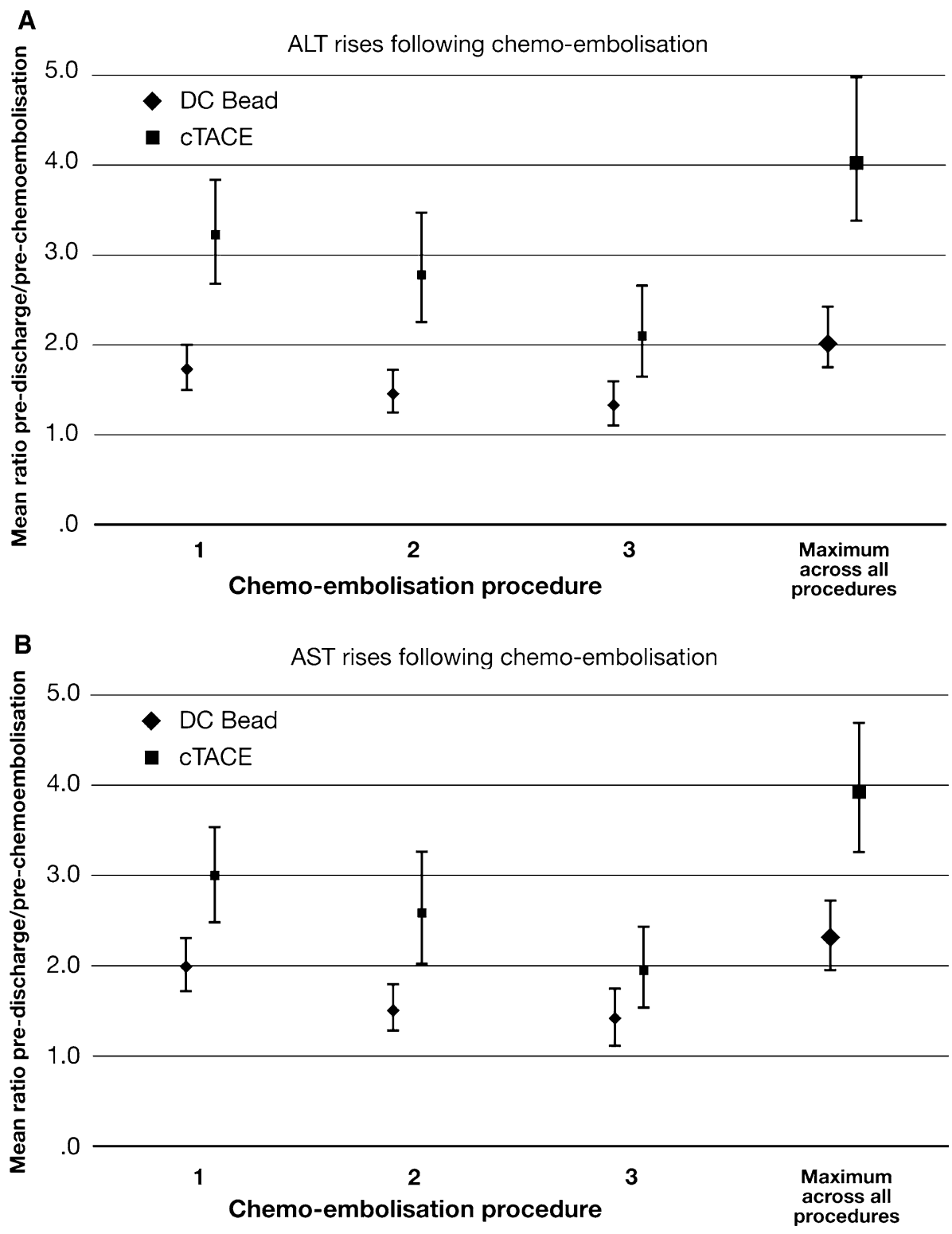

enabling the physician to safely deliver higher doses of doxorubicin compared to cTACE.

Post hoc analyses of the predefined stratified groups of higher-risk patients demonstrated that DC Bead provided significant advantages in treating patients with more advanced disease, where improved response and disease control and good tolerability were achieved. This finding is of particular significance for Child-Pugh B and ECOG 1 patients, for whom treatment with chemoembolization has been controversial [6]. Contrary to the observation in the cTACE arm, the response rate for DC Bead in these subgroups was maintained. This suggests that the improved tolerability of DC Bead allows treatment to be repeated according to planned schedule even in these more vulnerable patients. These favorable results establish DC Bead as a viable embolic agent for studies evaluating the combination of TACE with systemic administration of targeted agents such as Sorafenib, in intermediate HCC. In advanced HCC, Sorafenib has shown a survival benefit with low radiological response rates [27-29].

A limitation of the current study is that the number of patients required to show statistically significant superiority was underestimated due to the higher response rate of cTACE (44\%) compared with the original assumption (35\%) [16]. Robust time-to-progression and survival data would require further randomized controlled trials with longer follow-ups.

In conclusion, TACE with DC Bead and doxorubicin is safe and effective in the treatment of intermediate-stage HCC and offers benefit to patients with more advanced disease. 
Table 3 Effects of systemic doxorubicin (safety population)

\begin{tabular}{|c|c|c|c|c|}
\hline \multirow{2}{*}{$\begin{array}{l}\text { Event/SWOG } \\
\text { toxicity grade }\end{array}$} & \multicolumn{2}{|c|}{ DC Bead $(n=93)$} & \multicolumn{2}{|c|}{$\operatorname{cTACE}(n=108)$} \\
\hline & $\begin{array}{l}\text { No. of } \\
\text { events }\end{array}$ & $\begin{array}{l}\text { No. of } \\
\text { patients }\end{array}$ & $\begin{array}{l}\text { No. of } \\
\text { events }\end{array}$ & $\begin{array}{l}\text { No. of } \\
\text { patients }\end{array}$ \\
\hline Alopecia & 1 & $1(1.1 \%)$ & 23 & $22(20.4 \%)$ \\
\hline Grade 1 & 1 & & 12 & \\
\hline Grade 2 & 0 & & 11 & \\
\hline Marrow suppression & 5 & $5(5.4 \%)$ & 8 & $6(5.6 \%)$ \\
\hline Grade 1 & 2 & & 1 & \\
\hline Grade 2 & 2 & & 1 & \\
\hline Grade 3 & 1 & & 4 & \\
\hline Grade 4 & 0 & & 2 & \\
\hline Mucositis & 4 & $4(4.3 \%)$ & 7 & $6(5.6 \%)$ \\
\hline Grade 1 & 4 & & 5 & \\
\hline Grade 2 & 0 & & 1 & \\
\hline Grade 3 & 0 & & 1 & \\
\hline Skin discoloration & 2 & $2(2.2 \%)$ & 2 & $2(1.9 \%)$ \\
\hline Grade 1 & 1 & & 0 & \\
\hline Grade 2 & 1 & & 2 & \\
\hline
\end{tabular}

Note: Serious adverse events were defined as events (1) resulting in death, (2) that were immediately life-threatening, (3) resulting in permanent or significant disability/incapacity, or (4) requiring or extending inpatient hospitalization or (5) congenital anomaly/birth defects

Acknowledgments The study was sponsored by Biocompatibles UK Ltd. The authors acknowledge the contributions of Maria Schoder, Martin Funovics, Christian Loewe, Johanna Moyses, Dimitrios Kelekis, Nikolaos Kelekis, Alexis Kelekis, Emmanouil Emmanouil, Roman Klöckner, Christoph Düber, Gerd Otto, Marcus-Alexander Wörns, Verena Khan, Renate Hammerstingl, Clara Lee, Kathrin Eichler, Olivier Guillaud, Sabine Ficarelli, Nicolas Mennesson, Nathalie Rauscher, Beat Müllhaupt, Dominik Weishaupt, Pietro Majno, Laurent Spahr, Christoph D. Becker, Thierry de Baere, Frederic Deschamps, Pramod Roo, Vlad Ratzui, Phillipe Clluzel, Sebastien Novellas, Albert Tran, Denis Ouzan, Jean Gugenheim, Tim Greten, Timm Kirchhoff, Herbert Rosenthal, Joachim Lotz, Valerie Vilgrain, Annie Sibert, Peter Huppert, Hubertus Wietholtz, A Limmer, Pillippe Otal, Francis Joffre, Julien Auriol, Jean Marie Péron, Peter Waldenberger, Andreas Chemelli, Ivo Graziadei, Juergen Triller, and Ralph Kickuth ( $n=52$; order based on site recruitment).

\section{Appendix 1}

Austria

\section{Medical University of Vienna}

Department of Cardiovascular and Interventional Radiology: Johannes Lammer, Herbert Langenberger, Maria Schoder, Martin Funovics, Christian Loewe, Johanna Moyses

Department of Surgery: Thomas Grünberger
Department of Hepatology \& Gastroenterology: Christian Müller

\author{
Medical University Innsbruck \\ Department of Radiology: Peter Waldenberger, Andreas \\ Chemelli \\ Department of Gastroenterology \& Hepatology: Ivo \\ Graziadei
}

Germany

Goethe University, Frankfurt

Department of Radiology: Thomas Vogl, Verena Khan, Renate Hammerstingl, Clara Lee, Kathrin Eichler

Guttenberg University, Mainz

Department of Radiology: Michael Pitton, Roman Klöckner, Christoph Düber, Gerd Otto

Department of Internal Medicine: Marcus Schuchmann,

Marcus-Alexander Wörns

Medizinische Hochschule Hannover

Tim Greten, Timm Kirchhoff, Herbert Rosenthal, Joachim Lotz

Klinikum Darmstadt

Department of Radiology: Peter Huppert

Department of Gastroenterology: Hubertus Wietholtz, A. Limmer

France

Hospices Civils de Lyon, CHU, Hopital Edouard Herriot Department of Gastrointestinal Imaging: Frank Pilleul, Sabine Ficarelli, Nicolas Mennesson, Nathalie Rauscher Department of Hepatogastroenterology: Jerome Dumortier, Olivier Guillaud

Hôpital Beaujon, Clichy

Valerie Vilgrain, Annie Sibert

Hôpital Archet II, Nice

Department of Radiology: Patrick Chevallier, Sebastien Novellas, Albert Tran, Denis Ouzan, Jean Gugenheim Hôpital Huriez, Lille

Department of Radiology: Géraldine Sergent

CHU Rangueil, Toulouse

Department of Radiology: Pillippe Otal, Francis Joffre, Julien Auriol

\section{Hôpital Purpan}

Service d'Hépato-Gastro-Entérologie: Jean Marie Péron Hôpital La Pitiè Salpetrière, Paris

Service d'Hépato-Gastroentérologie: Yves Benhamou, Vlad Ratzui, Phillipe Clluzel 
Institut Gustave Roussy, Villejuif

Department of Radiology: Thierry de Baere, Frederic

Deschamps, Pramod Roo

Hôpital Paul Brousse, Paris

Yves Ajavon, Sameh Awad, Marie-France Bellin, Didier Samuel, Denis Castaing, Rene Adam, Jean-Charles Duclos Vallee, Faouzy Saliba, Daniel Azoulay

\section{Switzerland}

\section{CHU Vaudois, Lausanne}

Department of Radiology: Alban Denys

Inselspital, Bern

Department of Radiology: Juergen Triller, Ralph Kickuth

\section{Hopitaux Universitaires de Geneve}

Department of Radiology: Sylvain Terraz, Christoph D. Becker

Department of Surgery: Pietro Majno

Department of Gastroenterology and Hepatology: Laurent Spahr

Universitaetsspital Zuerich

Department of Radiology: Thomas Pfammatter, Dominik Weishaupt

Clinic of Gastroenterology \& Hepatology: Beat Muellhaupt

\section{Greece}

\section{Eugenidion Hospital, University of Athens}

Second Department of Radiology: Katerina Malagari, Pomoni Maria, Dimitrios Kelekis, Nikolaos Kelekis, Alexis Kelekis, Emmanouil Emmanouil

\section{References}

1. Altekruse SF, McGlynn KA, Reichman ME (2009) Hepatocellular carcinoma incidence, mortality, and survival trends in the United States from 1975 to 2005. J Clin Oncol 27:1485-1491

2. Höpfner M, Schuppan D, Scherübl H (2008) Growth factor receptors and related signalling pathways as targets for novel treatment strategies of hepatocellular cancer. World J Gastroenterol 14:1-14

3. Llovet JM, Bru C, Bruix J (1999) Prognosis of hepatocellular carcinoma: The BCLC staging classification. Semin Liver Dis 19:329-338

4. Bruix J, Llovet JM (2002) Prognostic prediction and treatment strategy in hepatocellular carcinoma. Hepatology 35:519-524

5. O'Neil BH, Venook AP (2007) Hepatocellular carcinoma: the role of the North American GI Steering Committee Hepatobiliary Task Force and the advent of effective drug therapy. Oncologist 12:1425-1432
6. Bruix J, Sherman M (2005) Practice Guidelines Committee, American Association for the Study of Liver Diseases. Management of hepatocellular carcinoma. Hepatology 42:1208-1236

7. Gish RG, Porta C, Lazar L et al (2007) Phase III randomized controlled trial comparing the survival of patients with unresectable hepatocellular carcinoma treated with nolatrexed or doxorubicin. J Clin Oncol 25:3069-3075

8. Llovet JM, Ricci S, Mazzaferro V et al (2008) Sorafenib in advanced hepatocellular carcinoma. N Engl J Med 359:378-390

9. Raoul JL, Heresbach D, Bretagne JF et al (1992) Chemoembolisation of hepatocellular carcinomas. A study of the biodistribution and pharmacokinetics of doxorubicin. Cancer 70:585-590

10. Lin DY, Liaw YF, Lee TY, Lai CM (1988) Hepatic arterial embolisation in patients with unresectable hepatocellular carcinoma - a randomised controlled trial. Gastroenterology 94:453456

11. Group d'Etude et de Traitment du Carcinome Hépatocellulaire (1995) A comparison of lipiodol chemoembolisation and conservative treatment for unresectable hepatocellular carcinoma. $\mathrm{N}$ Engl J Med 332:1256-1261

12. Raoul JL, Guyader D, Bretagne JF et al (1997) Prospective randomized trial of chemoembolisation versus intra-arterial injection of ${ }^{131}$ I-labeled-iodized oil in the treatment of hepatocellular carcinoma. Hepatology 26:1156-1161

13. Bruix J, Llovet JM, Castells A et al (1998) Transarterial embolisation versus symptomatic treatment in patients with advanced hepatocellular carcinoma: results of a randomised, controlled trial in a single institution. Hepatology 27:1578-1583

14. Pelletier G, Ducreux M, Gay F et al (1998) Treatment of unresectable hepatocellular carcinoma with lipiodol chemoembolisation: a multicentre randomised trial. J Hepatol 29:129-134

15. Lo CM, Ngan H, Tso WK et al (2002) Randomised controlled trial of transarterial lipiodol chemoembolisation for unresectable hepatocellular carcinoma. Hepatology 35:1164-1171

16. Llovet JM, Real MI, Montana X et al (2002) Arterial embolisation or chemoembolisation versus symptomatic treatment in patients with unresectable hepatocellular carcinoma: a randomised controlled trial. Lancet 359:1734-1739

17. Llovet JM, Bruix J, for the Barcelona-Clínic Liver Cancer Group (2003) Systematic review of randomized trials for unresectable hepatocellular carcinoma: chemoembolisation improves survival. Hepatology 37:429-442

18. Marelli L, Stigliano R, Triantos C et al (2007) Transarterial therapy for hepatocellular carcinoma: Which technique is more effective? A systematic review of cohort and randomized studies. Cardiovasc Interv Radiol 30:6-25

19. Lewis AL, Gonzales MV, Lloyd AW et al (2006) DC Bead: in vitro characterisation of a drug-delivery device for transarterial chemoembolisation. J Vasc Interv Radiol 17:335-342

20. Lewis AL, Gonzalez MV, Leppard SW et al (2007) Doxorubicin eluting beads. 1. Effects of drug loading on bead characteristics and drug distribution. J Mater Sci Mater Med 18:1691-1699

21. Lewis AL, Taylor RR, Hall B et al (2006) Pharmacokinetic and safety study of doxorubicin-eluting beads in a porcine model of hepatic arterial embolisation. J Vasc Interv Radiol 17:1335-1343

22. Hong K, Khwaja A, Liapi E et al (2006) New intra-arterial drug delivery system for the treatment of liver cancer: preclinical assessment in a rabbit model of liver cancer. Clin Cancer Res 12:2563-2567

23. Varela M, Real MI, Burrel M et al (2007) Chemoembolisation of hepatocellular carcinoma with drug eluting beads: efficacy and doxorubicin pharmacokinetics. J Hepatol 46:474-481

24. Poon RT, Tso WK, Pang RW et al (2007) A phase I/II trial of chemoembolisation for hepatocellular carcinoma using a novel intra-arterial drug-eluting bead. Clin Gastroenterol Hepatol 5:1100-1108 
25. Kettenbach J, Stadler A, Katzler IV et al (2008) Drug-loaded microspheres for the treatment of liver cancer: review of current results. Cardiovasc Interv Radiol 31:468-476

26. Malagari K, Chatzimichael K, Alexopöulou E et al (2008) Transarterial chemoembolisation of unresectable hepatocellular carcinoma with drug eluting beads: results of an open-label study of 62 patients. Cardiovasc Interv Radiol 31:269-280

27. Llovet JM, Di Bisceglie AM, Bruix J, Panel of Experts in HCCDesign Clinical Trials et al (2008) Design and endpoints of clinical trials in hepatocellular carcinoma. J Natl Cancer Inst 100:698-711

28. Moher D, Schulz KF, Altman DG (2001) The CONSORT statement: revised recommendations for improving the quality of reports of parallel-group randomised trials. Lancet 357:1191-1194

29. Hopewell S, Clarke M, Moher D et al (2008) CONSORT Group. CONSORT for reporting randomised trials in journal and conference abstracts. Lancet 371:281-283
30. Bruix J, Sherman M, Llovet JM et al (2001) Clinical management of hepatocellular carcinoma. Conclusions of the Barcelona-2000 EASL conference. European Association for the Study of the Liver. J Hepatol 35:421-430

31. Wang SK, Tsiatis AA (1987) Approximately optional oneparameter boundaries for group sequential trials. Biometrics 43:193-199

32. Satake M, Uchida H, Arai Y et al (2008) Transcatheter arterial chemoembolization (TACE) with lipiodol to treat hepatocellular carcinoma: survey results from the TACE study group of Japan. Cardiovasc Interv Radiol 31:756-761

33. Grosso M, Vignali C, Quaretti P et al (2008) Transarterial chemoembolization of hepatocellular carcinoma with drug-eluting microspheres: preliminary results from an Italian multicenter study. Cardiovasc Interv Radiol 31:1141-1149 